\title{
Daily Activity Recognition with Inertial Ring and Bracelet: an Unsupervised Approach
}

\author{
Alessandra Moschetti, Laura Fiorini, Dario Esposito, Paolo Dario, Member, IEEE and Filippo Cavallo, \\ Member, IEEE
}

\begin{abstract}
Daily activity recognition can help people to maintain a healthy lifestyle and robot to better interact with users. Robots could therefore use the information coming from the activities performed by the users to give them some custom hints to improve lifestyle and daily routine. The pervasiveness of smart things together with the advances in cloud robotics can help the robot to perceive and collect more information about the users and the environment. In particular thanks to the miniaturization and low cost of Inertial Measurement Units, in the last years, body-worn activity recognition has gained popularity. In this work, we investigated the performances of an unsupervised machine learning algorithm to recognize eight different gestures performed in daily living wearing a system composed by two inertial sensors placed on the hand and on the wrist. In this context our aim is to evaluate whether the system is able to recognize the gestures in more realistic applications, where is not possible to have a training set. The classification problem was analyzed using an unsupervised approach (k-mean), with an intra-subject and an inter-subject analysis, and two supervised approaches (Support Vector Machine and Random Forest) with a 10-fold cross validation analysis and with a Leave-One-Subject-Out to compare the results. The outcomes show that even in an unsupervised context the system is able to recognize the gestures with an averaged accuracy of 0.86 in the inter-subject approach.
\end{abstract}

\section{INTRODUCTION}

Maintaining a healthy lifestyle is becoming more important, due to the increase in the number of persons with chronic diseases [1]. In this context, technological solutions, and robot among others, can help to follow this objective by suggesting a healthy way of living and controlling the daily behavior [2]. Particularly, robots could interact properly with the user, personalizing the interaction, becoming thus more like a companion [3]. Particularly, by using this information, domestic robot could suggest some tailored hints to improve the human-beings lifestyle and to identify any behavioral changes. In effect, recent study demonstrate correlation between the alteration in daily lifestyle of elderly people and their cognitive status [4]

Nowadays, the advances in Internet of Things [5] and cloud robotics [6] extend companion robot abilities in terms of perception of surrounding environments and computing abilities respectively. Additionally, compared to last years,

\footnotetext{
*Research supported by the European Community's Seventh Framework under grant agreement No. 601116 (Echord++ project).

A.Moschetti, L. Fiorini, D. Esposito, P. Dario and F. Cavallo are with the BioRobotics Institute, Scuola Superiore Sant'Anna Viale Rinaldo Piaggio, 34, Pontedera 56025, Italy; (Contact Information: +39-0587-672152; email: a.moschetti@sssup.it, 1.fiorini@sssup.it, d.esposito@sssup.it , paolo.dario@sssup.it and f.cavallo@sssup.it)
}

the technological progress has made these service robots more common in our daily life [7]. Thanks to these advances, the robot has the possibility to collect more information from the user and, at the same time, to be aware of the context, increasing therefore the knowledge of the robot about what is around it [8]. In effect, one of the main advantages of cloud robotics approach rely on the exploitation of remote machine learning abilities to analyze data [9]. In this context, the robot could be aware of what the user is doing without the need to be in the same environment and interact with him/her only in case of need.

Roboticist and research team are in front of new scientific challenges [10] "How could we aggregate all this information? How can we use it to interpret the human behavior?". Therefore, in this context, research on activity recognition plays an important role in monitoring people and in understanding their daily routine. Among the others, the activities of daily living are important to be recognized, especially speaking about old persons, whose number is increasing with respect to the population worldwide [11]. For instance, people with cognitive disorders have problems in drinking and eating activities or in reminding tasks [12].

As stated from the literature evidences, these activities can be recognized using external or wearable sensors [13]. In the first case, sensors are included in the environment [14] or vision systems are used to infer the activity that is performed [15]. However this system could present some limitations due to privacy, in case of video analysis [16] and to the need to interact with specific landmarks in case of environmental sensors. In the second case, activities are inferred by the movement made by the users. Thanks to the miniaturization of Inertial Measurement Units (IMUs), wearable sensors have been used for activity recognition tasks, allowing to identify different daily activities. During the past, several studies had been conducted with wearable sensors placed in different part of the body [13]. However, to make this possible, it is necessary not only to have unobtrusive wearable sensors that do not interfere with the activities to be performed, but to identify a minimal set of wearable sensors which optimize the recognition performance [17]. Additionally, to make the system closer to a real case, pattern learning algorithms should be able to operate without labelled data, as it is too resource intensive for someone to verify the large quantities of data that is generated in a real living environment [18].

This work is therefore focused on the application of unsupervised machine learning algorithms to recognize gestures that can be used to infer activities of daily living. Starting from our previous work presented in [19], we proposed the analysis of the performances of a unsupervised technique applied to the recognition of different daily 
gestures, including eating and drinking ones, with a minimal combination of wearable sensors.

\section{RELATED WORKS}

Over the last years, wearable sensors and in particular IMUs have been used in several activity recognition works, thanks to the miniaturization and affordability of these sensors [17]. Several works have been presented where IMUs were used for activity recognition problems, analyzing also the best placement on human body [20]. Among the different placement, due to the presence of these sensors in commercial devices such as smartphones and wrist-worn devices, the ability of IMUs placed on the wrist and in the pocket to discriminate among different activities has been investigated. In particular, Guiry et al [21] analyzed the use of a smartwatch and a smartphone using both IMUs and other sensors on the devices, like pressure and light sensors and GPS. They adopted five different supervised machine learning approaches (decision tree, both C4.5 and CART, Naïve Bayes, Multi-Layer Perceptrons and Support Vector Machine) to train and test the dataset, combining data coming from the sensors. In particular in case of the smartwatch only the recognition rate reached $89 \%$ to recognize activities such as walking, sitting, lying, standing. Nevertheless, even if the recognition rate reached high value, the considered activities did not involve the use of the hand and the authors performed only supervised learning analyses.

Mortazavi et al. [22] compared the recognition ability of smartphones and smartwatches considering the IMU on them- Using a Support Vector Machine approach to recognize activities like sitting, standing, lying, walking and transitions, the F-measure reached 0.930 considering the smartwatch only. However, even in this work, only supervised machine learning algorithm was considered to recognize activities where the hand was not included.

Weiss et al. [23] analyzed how a smartwatch could recognize different kind of activities that go from walking to eating using five different machine learning algorithms (Random Forest, J48 decision tree, Naïve Bayes, Multi-layer perceptron and IB3 instance-based learning). In this work, they showed that the eating activities did not have good recognition rate compared to activities like walking, sitting and standing, reaching an overall accuracy of 0.703 .

As stated from the state of the art, the wrist is a good location for wearable sensors, due to the low obtrusiveness, but it does not give information about the fingers movements. Consequently, we decided to place some sensing units on the fingers in order to collect data useful to discriminate among gestures that involve also the use of the hand. In our previous work [19], we investigated how the recognition rate of different activities vary combining different sensors on the hand and on the wrist. In particular, the results of this previous study demonstrated that the combination of inertial sensors placed on the index finger and on the wrist can be used to recognize different daily gestures, such as eating and drinking with different tools and performing acts of some personal hygiene. With this configuration, in fact, we reached good value of F-measure (0.884) and accuracy (0.890) compared with the wrist sensor only $(0.622$ and 0.650 respectively). Best results were obtained with the use of a sensor even on the thumb (F-measure equal to 0.908 and accuracy equal to 0.913 ), but the index-wrist combination was the one that showed the best trade-off between recognition rate and obtrusiveness.

In our previous work, we used supervised machine learning algorithms that, also according to the state of the art, show in general promising results, but they need to have training dataset made of labelled data, which can be difficult to be generated. Moreover in case of added activities it is necessary to build the dataset again and the model needs to be retrained [18].

Aim of this work is therefore to investigate the use of an unsupervised machine learning algorithm to recognize eight daily gestures. In particular, starting from the results presented in our previous work, where it was shown that the use of a sensor on the finger improves the recognition of gesture involving the hand, in the presented paper, we analyze the performances of an unsupervised machine learning algorithm comparing them with the ones of two supervised ones. The aim of this work is therefore to investigate the use in more realistic application of this sensor configuration, considering that unsupervised machine learning algorithms do not require labelled data, thus avoiding the need of a training dataset, and can therefore be more adaptable to real application of human activity recognition [24].

The rest of this paper is structured as follows. In Sect. III, we detail the applied methodology used in this research. Particularly we present the system architecture and data analysis performed. Section IV summarizes and discusses the results and Sect. V concludes the paper.

\section{Methods}

According to the results described in our previous work [19], we decided to start the analysis presented in this paper considering only the index and the wrist sensors configuration.

In this paragraph, we described the sensors used, the acquisition of the data and the analysis carried out.

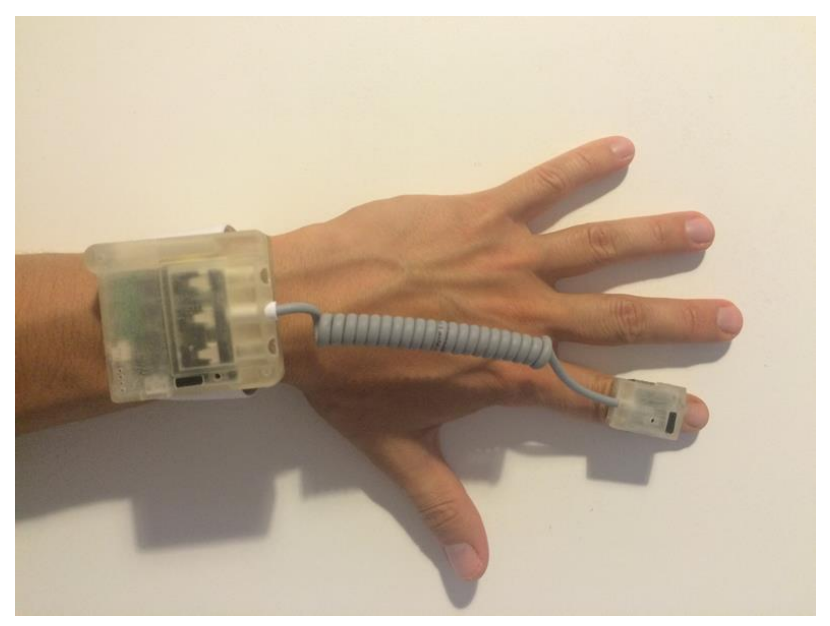

Figure 1 - Inertial Sensor Units and placement on the user 
TABLE I

GESTURE DESCRIPTION

\begin{tabular}{ll}
\hline \hline \multicolumn{1}{c}{ Gesture } & \multicolumn{1}{c}{ Description } \\
\hline $\begin{array}{l}\text { HA: Eating with the hand } \\
\text { GL: Drinking with a glass }\end{array}$ & $\begin{array}{l}\text { Users took the food with the hand, moved it to the mouth and back to the table } \\
\text { Persons were asked to grasp the glass, move it to the mouth and then leave it on the table }\end{array}$ \\
FK: Eating with a fork & $\begin{array}{l}\text { Participants had to take a piece of already cut fruit with the fork, eat it and then move the hand back to } \\
\text { the table without leaving the fork } \\
\text { Persons had to use the spoon, load it with some yogurt, move it to the mouth and then back to the table } \\
\text { without leaving it }\end{array}$ \\
SP: Eating with a spoon & $\begin{array}{l}\text { Users were asked to grasp the mug, move it to the mouth and then back on the table, leaving it } \\
\text { CP: Drinking with a cup }\end{array}$ \\
$\begin{array}{l}\text { Participants had to take the phone, move it to the head and back on the table after a few seconds } \\
\text { TB: Brushing the teeth with a toothbrush }\end{array}$ & $\begin{array}{l}\text { Persons were asked to take the toothbrush from the sink, move it to the mouth to brush the teeth and } \\
\text { back on the sink } \\
\text { The gesture consisted in taking the hairbrush from the sink, moved it to the head, used it two/three } \\
\text { times and back to the sink }\end{array}$ \\
\hline \hline
\end{tabular}

\section{A. System Descriptions}

The system used for the data acquisition consisted of two sensor units, each integrating a INEMO-M1 board with dedicated microcontroller (ARM 32-bit Cortex ${ }^{\mathrm{TM}}-\mathrm{M} 3 \mathrm{CPU}$, STMicroelectronics). Each board consists of a LSM303DLHC (6-axis geomagnetic module, STMicroelectronics) and L3G4200D (3-axis digital gyroscope, STMicroelectronics) and I2C digital output. A quaternion-based Kalman filter, adapted from [25], is implemented on each unit and the Euler Angles are evaluated so to collect the orientation of the modules, beyond the acceleration and the angular velocity. Data are collected at $50 \mathrm{~Hz}$. More detailed information about the sensor units can be found in [19].

Accelerometer and gyroscope data were filtered on board with a fourth order low pass filter with a cutoff frequency of $5 \mathrm{~Hz}$, to remove high frequency rumor and tremor [26]. Sensors were calibrated both in static and in dynamic condition in order to calculate the offset and the sensitivity that affect the system.

As shown in Fig. 1, sensors were placed on the intermediate phalange of the index and on the wrist of the user (like a Smartwatch). Both the devices were placed on the dominant arm of the user.

\section{B. Experimental Setting}

Users were asked to perform eight different gestures, chosen to identify different activities of daily living, such as feed and personal hygiene. The chosen gestures are described in Table I. Ten young participants (6 females and 4 males, whose ages ranged from 21 to $34(29.1 \pm 3.9))$ were asked to perform the chosen gestures in the DomoCasa Lab, which is a $200 \mathrm{~m} 2$ fully furnished apartment located in Peccioli, Pisa (Echord ++ RIF). Using this location permitted to limit as much as possible unnatural movements linked to a laboratory setting, thanks to the realistic environment.

Participants were asked to perform each gesture 40 times continuously, without any constriction in the way the movements have to be performed. At the beginning of each sequence users were asked to keep the hand and the forearm still for few seconds on a flat surface in order to calibrate each session and calibrate the sensors position with respect to the first gesture made (HA). During the sequence, users were observed and at the beginning and at the end of each of the
40 gestures a label was put in order to identify the gestures in the acquisition. In Fig. 2 a focus on the grasping of different objects is shown.

\section{Data Analysis}

In order to achieve the proposed goals, two different data analysis procedures were performed. The first analysis aimed to cluster the gestures with an unsupervised approach. Whereas, the second one aimed to compare these outcomes with two traditional inter-subject supervised approaches.

Firstly, the signals were segmented according to the labels and processed to extract the significant features [17], shown in Table II. The final dataset included 3200 gestures with a total of 44 features ( $(12$ accelerometers +10 angles) $\mathrm{x}$ 2) labelled with the corresponding gesture. The matrix of the features was normalized using a Z-norm in order to avoid distortion.

Unsupervised machine learning clustering technique is used to grouped the performed gestures into cluster. Particularly, in this work, K-Means (KM) algorithm was applied considering the Euclidian distance, additionally, in order to avoid local minima we considered the replicate strategy (in this study we considered 5 replicates). We had chosen this algorithm because it is the most widely used unsupervised machine learning techniques [27] Silhouette index [28] was computed to assess the cluster validity and to quantitatively analyze the clustering process. A higher value of this index indicates that the clusters are compact and well separated.

In order to evaluate the robustness of the system and how concretely it could recognize gestures performed by

TABLE II

SigNIFICANT FEATURES

\begin{tabular}{cc}
\hline \hline Data & Extracted Features \\
\hline \multirow{3}{*}{ Accelerometer $(\mathrm{x}, \mathrm{y}, \mathrm{z})$} & Mean \\
& Standard Deviation \\
& Root Mean Square \\
& Mean Absolute Deviation \\
\hline \multirow{3}{*}{ Roll and Pitch angles } & Mean \\
& Standard Deviation \\
& Mean Absolute Deviation \\
\hline \hline
\end{tabular}




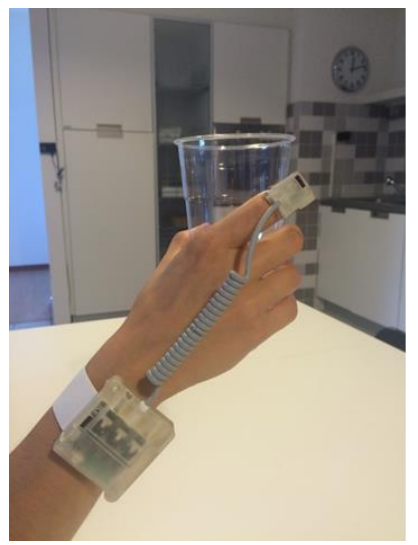

(a)

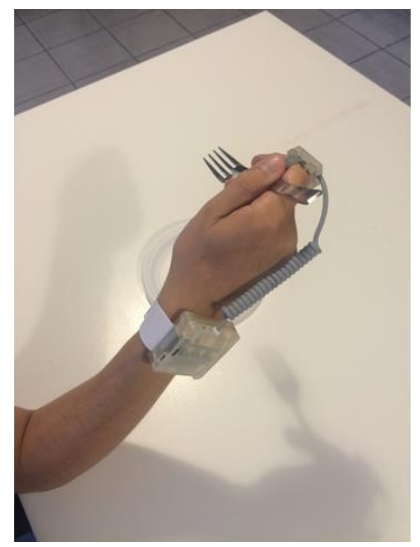

(b)

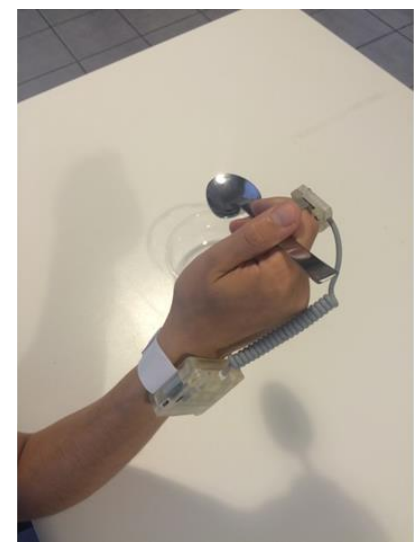

(c)

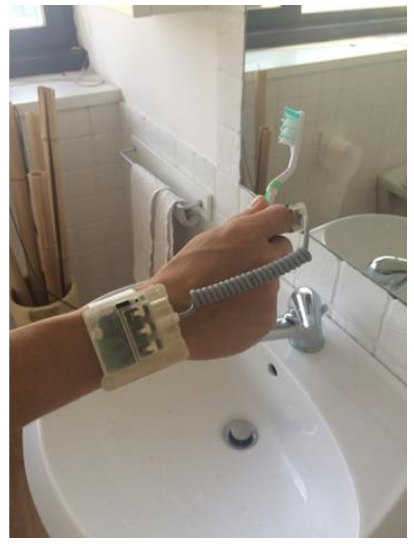

(d)

Figure 2 - Example of grasping of different objects: (a) taking a glass, (b) grasping a fork, (c) grasping a spoon and (d) taking the toothbrush.

same/different users, intra- and inter-subject analysis was then performed. In the first analysis we considered the gestures performed by a single subject, in the second one we considered the totality of the dataset. The performances were evaluated with an external criteria [29] by comparing the output with our "a-priori" knowledge.

As concern the supervised analysis, two different machine learning algorithms were considered among the ones that are usually used in activity recognition problems. In particular, we applied the Support Vector Machine (SVM) algorithm, as described in [19] and the Random Forest algorithm. The second one is an ensemble learning algorithm that builds a number of decision trees in the training phase and in classification gives as an output the mode of the classes of each trees. Each tree is built on a random selection of features and in the classification phase each tree receives the input vector and the output of the whole classifier is the maximum voting among the trees $[30,31]$. To analyze the data, we used the package for Matlab ${ }^{\circledR}$ developed by Abhishek Jaiantilal, which is based on the Breiman et al. algorithm [32]. All the options were set to default.

To evaluate the recognition accuracy of the system with the supervised machine learning algorithms, two kind of analysis were carried, that are k-fold cross validation and Leave-One-Subject-Out cross validation (LOSO). In the former we tested a 10 -fold cross validation $(10-\mathrm{fCV})$ on the complete dataset, while in the latter nine users were used as a training set and one as a test set, using all the users as test set in rotation.

The results obtained with the unsupervised and supervised machine learning algorithms were presented as a confusion matrix. Then, overall accuracy, F-measure, precision, recall and specificity were computed as described in [17] in order to compare and discuss the performance of this two approaches.

\section{EXPERIMENTAL RESULTS}

The main goal of this study was to evaluate whether an unsupervised machine learning approach was suitable for the recognition of the selected activities. Data coming from the proposed system configuration (inertial sensors placed on the wrist and the index finger) were analyzed and used as database for an unsupervised algorithm. Precision, Accuracy, Specificity, Recall and F-Measure were computed for both approaches in order to compare the results.

As regard the unsupervised analysis, the results of the intra-subject analysis, obtained as the mean value of the 10 subject-dataset, are comparable with the results of the supervised analysis conducted with the 10-fold cross validation approaches. Particularly, Precision, Accuracy, Specificity, Recall and F-Measure are $>0.99$ for both approaches (see Tab.III). The unsupervised analysis showed that the eight gestures are performed in a different way by the user and the system is therefore very good in discriminating among the gestures made by the same user.

Considering the 10 -fold cross validation approach, the

TABLE III

RESULTS OF THE UNSUPERVISED AND SUPERVISED ANALYSIS

\begin{tabular}{cccccc}
\hline \hline Algorithm & Precision & Recall & Specificity & Accuracy & F-Measure \\
\hline Unsupervised & & & & 0.860 & 0.870 \\
Inter-Subject & 0.880 & 0.860 & 0.978 & 0.989 & 0.990 \\
Intra-Subject & 0.991 & 0.989 & 0.998 & 0.994 & 0.980 \\
Supervised & & & & 0.963 & 0.966 \\
SVM-10-fCV & 0.994 & 0.994 & 0.999 & 0.995 & 0.995 \\
SVM - LOSO & 0.970 & 0.963 & 0.994 & 0.985 & 0.985 \\
RF-10-fCV & 0.995 & 0.995 & 0.998 & & \\
RF-LOSO & 0.987 & 0.985 & & & \\
\hline \hline
\end{tabular}




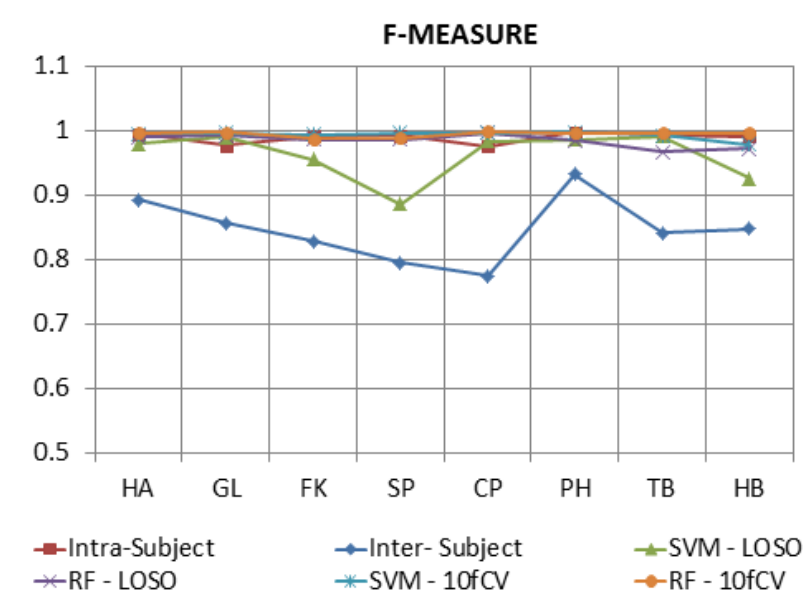

(a)

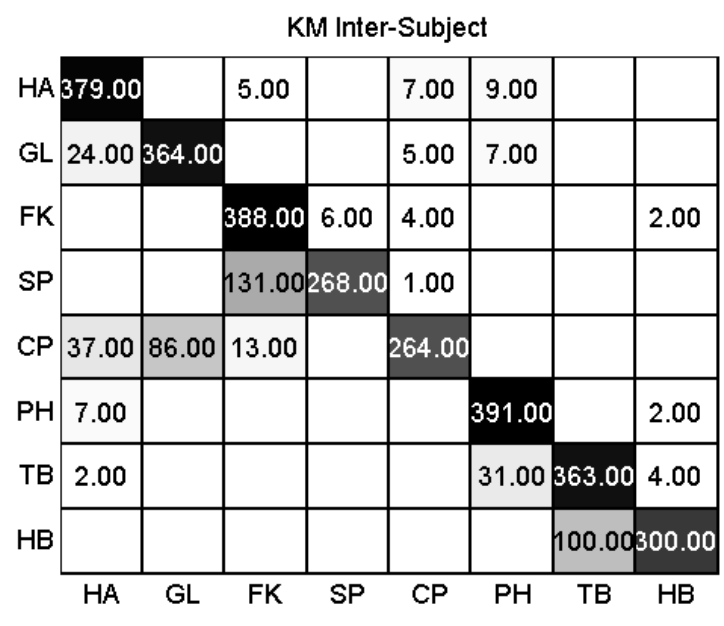

(b)

Figure 3 - (a) Comparison of the F-Measure metrics over the eight gestures in the supervised and in the unsupervised approaches (b) Confusion matrix

system is really good in recognizing the gestures, while in the LOSO approach, results are slightly lower, but still comparable for both algorithms. The F-measure is equal to 0.966 for the SVM and 0.985 for the RF, the accuracy in equal to 0.963 for the SVM and 0.985 for the RF (see Tab. III). This analysis investigate how the system is able to manage the unknown subjects and to quantify how the system is able to reject any disturbances due to differences in performing gestures of a specific subject in real cases. As confirmed by the high values, this system is robust in recognizing unseen subject' activities, demonstrating that the system is able to manage the differences among the users in performing the gestures. Similar results can be seen in the unsupervised inter-subject analysis that considers the variability of the subjects in performing the different activities and investigates the robustness of the system. The obtained results for this analysis are promising. Particularly, the F-measure is equal to 0.870 and the accuracy is equal to 0.860. As we can expect, these results are lower than the results of the intra-subject analysis because of the high variability between the subjects. The data were acquired with no restriction on the pose, the subjects were set free to perform the gesture as they want. Therefore, this results suggest that this system configuration is suitable for a future exploitation in real settings because the unsupervised approach is able to cluster the variable data with an high level of accuracy.

Additionally, Fig. 3.a reports the F-measure over the eight gestures. Particularly, in the inter-subject analysis the worst recognized gestures are $\mathrm{FK}, \mathrm{SP}$ and $\mathrm{CP}$ (F-measure equal to $0.828,0.795$ and 0.775 respectively). FK presents a precision of 0.723 and a high recall $(0.970)$, while SP and CP show high precision (0.978 and 0.940 respectively) and lower recall ( 0.670 and 0.660 respectively). In fact, as depicted in Fig. 3.b SP gesture is mainly confused with FK, and CP is confused with GL. SP/FK and CP/GL are very similar in terms of hand pose and are is therefore probable that the system confuses them. On the contrary, TB is the best recognized activity, 396 out of 400 gestures have been correctly classified, reaching a precision value of 0.893 and a recall of 0.977 .
As stated in the previous work, the addition of the index finger improved the recognition rate with respect to a single sensor on the wrist, considering that all the selected gestures involve the movement of the hand to the mouth/head. With respect to the state of the art the system is able to discriminate different gesture with improved results. The addition of the index sensor improve the recognition rate, which with a wrist sensor was very low in recognizing eating activities (accuracy equal to 0.527 and 0.627 for eating soup and drinking activities in case of an impersonal analysis [23]). In this work, we further investigate this configuration of sensors to evaluate the ability of the system to recognize the different gestures in case no training set is given obtaining good results in terms of accuracy and F-measure. The use of this configuration could be further investigate to evaluate the recognition rate in case of sitting, standing and lying activities that are often recognized with wrist sensors. In this way it could be possible to increase the number of recognized activities getting closer to real applications, thanks to the use of unsupervised machine learning algorithms.

\section{CONCLUSION}

The aim of this work was to investigate whether the system composed of a IMU placed on the index finger and on the wrist gave good results with unsupervised machine learning algorithms. The same dataset was used with unsupervised techniques and the results were compared with the supervised ones. In particular, the supervised algorithms gave good results, both in term of F-measure and accuracy $(>0.96)$ also in the LOSO analysis, showing a good ability in managing unknown users. The unsupervised algorithm gave also good results both in the intra-subject(F-measure $>0.99)$ and inter-subject $(\mathrm{F}$-measure $=0.87)$ analysis. These results are therefore promising for further investigation in real applications.

\section{ACKNOWLEDGMENT}

This work was supported by the European Community's 
Seventh Framework under grant agreement No. 601116 (Echord++ project).

\section{REFERENCES}

[1] R. Busse, "Tackling chronic disease in Europe: strategies, interventions and challenges (No. 20). WHO Regional Office Europe, 2010

[2] A. Moschetti, L. Fiorini, M. Aquilano, F. Cavallo, P. Dario, "Preliminary findings of the AALIANCE2 ambient assisted living roadmap". In Ambient Assisted Living; Springer International Publishing: Berlin, Germany, 2014; pp. 335-342

[3] N. Daosodsai and T. Maneewarn, "Fuzzy based emotion generation mechanism for an emoticon robot," Control, Automation and Systems (ICCAS), 2013 13th International Conference on, Gwangju, 2013, pp. 1073-1078.

[4] D. J. Cook, M. Schmitter-Edgecombe and P. Dawadi, "Analyzing Activity Behavior and Movement in a Naturalistic Environment Using Smart Home Techniques," in IEEE Journal of Biomedical and Health Informatics, vol. 19, no. 6, pp. 1882-1892, Nov. 2015.

[5] J. Cubo, A. Nieto, and E. Pimentel, 'A cloud-based Internet of Things platform for ambient assisted living.' Sensors , 2014, 14, pp. 1407014105

[6] B. Kehoe, S. Patil, P. Abbeel and K. Goldberg, "A Survey of Research on Cloud Robotics and Automation," in IEEE Transactions on Automation Science and Engineering, vol. 12, no. 2, pp. 398-409, April 2015.

[7] International Federation of Robotics, World Robotics 2015 Service Robots, available at: http://www.ifr.org/service-robots/statistics/].

[8] G. Hu, W. P. Tay and Y. Wen, "Cloud robotics: architecture, challenges and applications," in IEEE Network, vol. 26, no. 3, pp. 2128, May-June 2012.

[9] B. Kehoe, A. Matsukawa, S. Candido, J. Kuffner and K. Goldberg, "Cloud-based robot grasping with the google object recognition engine," Robotics and Automation (ICRA), 2013 IEEE International Conference on, Karlsruhe, 2013, pp. 4263-4270.

[10] M. Vrikkas, C. Nikou, and I.A: Kakadiaris, "A review on Human Activity Recognition Methods", Frontiers in Robotics and AI, 2015, 2(28)

[11] Eurostat - European social statistics Demographic projection (2013)

[12] S.H. Zarit, K.E. Reever and J. Bach-Peterson, "Relatives of the impaired elderly: correlates of feelings of burden.”, The gerontologist, 1980, 20(6), pp. 649-655.

[13] S. C. Mukhopadhyay, "Wearable Sensors for Human Activity Monitoring: A Review," in IEEE Sensors Journal, vol. 15, no. 3, pp. 1321-1330, March 2015.

[14] N. C. Krishnan \& D.J. Cook, “ Activity recognition on streaming sensor data. Pervasive and mobile computing", 2014, 10, 138-154

[15] J.K. Aggarwal and L. Xia, "Human activity recognition from 3d data: A review",. Pattern Recognition Letters, 2014, 48, 70-80

[16] K. Arning and M. Ziefle, "Get that Camera Out of My House! Conjoint Measurement of Preferences for Video Based Healthcare Monitoring Systems in Private a Public Places", Inclusive Smart Cities and e-Health. Springer International Publishing, 2015, pp. 152164

[17] O. D. Lara and M. A. Labrador, "A Survey on Human Activity Recognition using Wearable Sensors," in IEEE Communications Surveys \& Tutorials, vol. 15, no. 3, pp. 1192-1209, Third Quarter 2013.

[18] Y. Kwon, K. Kang, and C. Bae, “ Unsupervised learning for human activity recognition using smartphone sensors". Expert Systems with Applications, 2014, 41(14), 6067-6074

[19] A. Moschetti, L. Fiorini, D. Esposito, P. Dario and F. Cavallo, "Recognition of Daily Gestures with Wearable Inertial Rings and Bracelets". Sensors, 2016, 16(8), 1341

[20] F. Attal, S. Mohammed, M. Dedabrishvili, F. Chamroukhi, L. Oukhellou, Y. Amirat, "Physical human activity recognition using wearable sensors". Sensors 2015, 15, 31314-31338.

[21] J.J. Guiry, P. van de Ven, and J. Nelson, “ Multi-sensor fusion for enhanced contextual awareness of everyday activities with ubiquitous devices". Sensors 2014, 14, 5687-5701
[22] B. Mortazavi,E. Nemati, K. VanderWall, H.G. Flores-Rodriguez, J.Y.J. Cai,;J. Lucier, A. Naeim, M. Sarrafzadeh, "Can Smartwatches Replace Smartphones for Posture Tracking?" Sensors 2015, 15, 26783-26800.

[23] G. M. Weiss, J. L. Timko, C. M. Gallagher, K. Yoneda and A. J. Schreiber, "Smartwatch-based activity recognition: A machine learning approach," 2016 IEEE-EMBS International Conference on Biomedical and Health Informatics (BHI), Las Vegas, NV, 2016, pp. 426-429.

[24] D. Trabelsi, S. Mohammed, Y. Amirat and L. Oukhellou, "Activity recognition using body mounted sensors: An unsupervised learning based approach," The 2012 International Joint Conference on Neural Networks (IJCNN), Brisbane, QLD, 2012, pp. 1-7.

[25] S. Sabatelli, M. Galgani, L. Fanucci and A. Rocchi, "A Double-Stage Kalman Filter for Orientation Tracking With an Integrated Processor in 9-D IMU," in IEEE Transactions on Instrumentation and Measurement, vol. 62, no. 3, pp. 590-598, March 2013.

[26] E. Rovini, D. Esposito, C. Maremmani, P. Bongioanni, F. Cavallo, "Using Wearable Sensor Systems for Objective Assessment of Parkinson's Disease". In Proceedings of the 20th IMEKO TC4 International Symposium and 18th International Workshop on ADC Modelling and Testing Research on Electric and Electronic Measurement for the Economic Upturn, Benevento, Italy, 15-17 September 2014; pp. 15-17

[27] A.K. Jain, "Data clustering: 50 years beyond K-means", Pattern recognition letters, 2010, 31(8), 651-666.]

[28] P.J. Rousseeuw, " Silhouettes: a graphical aid to the interpretation and validation of cluster analysis", Journal of computational and applied mathematics, 1987, 20, 53-65

[29] A.K. Jain and R.C. Dubes, "Algorithms for clustering data”. PrenticeHall, Inc, 1988

[30] Z. Feng, L. Mo and M. Li, "A Random Forest-based ensemble method for activity recognition," 2015 37th Annual International Conference of the IEEE Engineering in Medicine and Biology Society (EMBC), Milan, 2015, pp. 5074-5077.

[31] L. Breiman, L, "Random forests." , Machine learning 45.1 (2001): 532

[32] https://code.google.com/archive/p/randomforest-matlab/ 